REGARDS

SUR LECONOMIE ALLEMANDE

BULLETIN ECONOMIQUE DU CIRAC
Regards sur l'économie allemande

Bulletin économique du CIRAC

$96 \mid 2010$

Varia

\title{
La formation continue en Allemagne
}

\section{Winfried Heidemann}

Traducteur : Isabelle Bourgeois

\section{OpenEdition}

\section{Journals}

Édition électronique

URL : http://journals.openedition.org/rea/4083

DOI : $10.4000 /$ rea.4083

ISBN : 978-2-8218-0888-1

ISSN : 1965-0787

Éditeur

CIRAC

Édition imprimée

Date de publication : 1 mai 2010

Pagination : 23-31

ISSN : 1156-8992

\section{Référence électronique}

Winfried Heidemann, «La formation continue en Allemagne », Regards sur l'économie allemande [En ligne], 96 | mai 2010, mis en ligne le 01 mai 2012, consulté le 04 mai 2019. URL : http:// journals.openedition.org/rea/4083 ; DOI : 10.4000/rea.4083 


\title{
La formation continue en Allemagne
}

\author{
Winfried Heidemann
}

En Allemagne, on compte d'innombrables institutions offrant de la formation continue, un large éventail de prestations dans des domaines les plus divers mais on y chercherait en vain un 'système cohérent' en la matière. Les entreprises s'impliquent dans la formation continue, les chambres consulaires assument elles aussi leurs responsabilités dans ce domaine, sans parler des universités populaires. La formation continue réglementée de perfectionnement (Aufstiegsfortbildung) côtoie le congé individuel de formation et l'offre de reclassement/reconversion des chômeurs. Aux côtés des dispositions légales ouvrant droit à congé de formation (Bildungsurlaub) et prévoyant un soutien financier pour les salariés (lois des Länder sur la formation continue, mesures de la politique pour l'emploi, lois et décrets sur la formation de perfectionnement), il y a les dispositions contractuelles: conventions conclues par les partenaires sociaux au niveau de la branche, accords internes à l'entreprise conclus par le Conseil d'établissement et la direction.

Or ce système très disparate dans sa diversité ne permet pas d'attester une participation durablement élevée des individus et des entreprises à la formation continue; en comparaison internationale, celle-ci est même nettement inférieure au taux recensés dans les autres grandes économies européennes. Est-ce à dire pour autant que la formation continue tiendrait en Allemagne une place relativement subalterne?

\section{Comment est réglementée la formation continue}

En Allemagne, la formation continue n'est que peu réglementée, bien qu'il existe, au niveau du Bund comme des Länder, une série de dispositions légales visant à promouvoir la formation continue. Ainsi, 12 des 16 Länder ont institué pour les salariés un droit à congé individuel de formation (Bildungsurlaub) : il est de cinq jours ouvrés par an, mais cette durée peut être cumulée à dix jours sur deux ans ; les salariés choisissent librement la formation à laquelle ils souhaitent participer parmi l'offre agréée par l'administration des Länder.

Alors qu'à l'origine, les formations agréées proposées dans le cadre de ce congé visaient exclusivement la culture générale et l'éducation politique, leurs contenus ont été recentrés ces dernières années sur les questions professionnelles. Pourtant, seul $1 \%$ environ des salariés recourt à ce congé individuel, pour de multiples raisons. La première invoquée est le manque de moyens financiers : le salarié est certes en droit de continuer à percevoir son salaire durant son congé de formation, mais les coûts de la formation sont à la charge des participants ou doivent être couverts grâce au soutien provenant d'autres sources. On suppose également, sous l'angle de l'économie de la formation cette fois, que étant donné que les formations sont essentiellement choisies en fonction des avantages professionnels qu'on en attend (salaire, carrière, meilleure exécution des tâches, sécurisation du poste de travail), l'offre des parcours réglementés, trop éloignés de la réalité de l'entreprise, n'est pas adaptée à cette demande. Enfin, on avance le manque de soutien apporté à ce type de formation par les employeurs et les problèmes rencontrés surtout dans les PME par les salariés désireux de bénéficier d'un tel congé individuel.

Le congé individuel est réglementé dans la plupart des Länder

Mais $1 \%$ seulement des salariés y recourent 
Formation de perfectionnement : lois-cadre du Bund pour les diplômes, organisation de leur délivrance par les chambres

La promotion de la formation continue des chômeurs relève du Bund

Régulation contractuelle de la formation au niveau de la branche ou de l'entreprise

Métallurgie, électrotechnique et chimie en pointe sur le congé individuel de formation
Cela dit, dans tous les Länder existent des programmes pour soutenir l'offre de formation et en garantir la qualité au sein du réseau des organisations de formation des adultes comme les universités populaires (Volkshochschulen).

En ce qui concerne la formation continue réglementée de perfectionnement offerte dans le prolongement d'une formation professionnelle initiale achevée, et qui mène le plus souvent au brevet de maître-artisan (Meister) ou de cadre spécialisé dans l'industrie et le commerce (Fachwirt), elle est réglementée par des lois-cadre fédérales fixant les critères de délivrance des diplômes. L'organisation de la certification et la délivrance des brevets de maîtrise et de Fachwirt relèvent quant à elles des chambres consulaires ( $\mathrm{CCl}$, chambres des métiers, etc.). Les salariés participant aux enseignements de préparation aux examens peuvent bénéficier d'un soutien financier en application de la loi fédérale relative à la promotion des formations de perfectionnement, dite "Meister-BAFöG ». Le même schéma s'applique à l'organisation des formations au diplôme de Techniker proposées par certaines Fachschulen, établissements d'enseignement professionnel, à la différence près que sa régulation relève des Länder.

Quant à la formation continue des chômeurs, sa promotion dans le cadre de la politique pour l'emploi relève du Bund. Au cours des dernières années, l'offre de formations qualifiantes sanctionnées par un diplôme a cependant été réduite, le placement rapide des chômeurs étant désormais la priorité. Enfin, dans le cadre de la politique de soutien à l'économie adoptée en réponse à la crise économique actuelle, les mesures de soutien à la formation continue dans l'entreprise, prévues par le dispositif du chômage partiel, ont été considérablement améliorées. L'expérience montre toutefois que les entreprises n'ont que très peu recouru aux possibilités existantes.

En Allemagne, les entreprises décident librement de l'offre comme de la mise en œuvre de la formation continue de leurs salariés. II n'existe aucune disposition ou obligation réglementaire en la matière. II existe en revanche une régulation-cadre contractuelle : dispositions prévues par les conventions de branche et les accords d'entreprise s'appliquant aux entreprises membres d'une organisation de branche ou aux établissements ayant un conseil d'établissement.

Aujourd'hui, un grand nombre de conventions tarifaires contiennent des dispositions relatives à la formation continue. Mais il convient de distinguer entre accords conclus au niveau de la branche et accords d'entreprise. Ces derniers ne s'appliquent qu'aux établissements de l'entreprise concernée; et lorsqu'ils contiennent des dispositions sur la formation continue, elles concernent le plus souvent l'organisation de la formation au sein de l'entreprise. Les conventions de branche, largement plus répandues en comparaison, se concentraient autrefois sur la mise à disposition, au sein de la branche, d'une offre de formation externe aux établissements ; c'était le cas du BTP ou du textile-habillement. Depuis peu, ces conventions contiennent également des dispositions relatives à l'organisation de la formation continue au sein de l'établissement. Priorité est alors donnée aux préconisations concernant l'identification des besoins en qualifications et la participation du Conseil d'établissement au processus de planification de la formation dans l'entreprise. Les conventions tarifaires conclues dans l'industrie métallurgique, électrotechnique et chimique au début des années 2000 ont joué un rôle de précurseur dans le domaine.

Dans l'industrie métallurgique et électrotechnique du Bade-Wurtemberg, une convention tarifaire entrée en vigueur en 2001 (ses principales dispositions ont été étendues depuis à toute l'Allemagne) a ouvert à chaque salarié le droit à dresser régulièrement avec son $\mathrm{DRH}$ le bilan de ses besoins personnels en matière de qualification, accompagné du droit à congé individuel de formation assorti de la garantie de l'emploi. Tous les ans, salariés et employeur effectuent conjointement le bilan des besoins en matière de formation ; et à l'issue des entretiens est conclue une convention individuelle de formation continue. Dans l'industrie chimique, une convention tarifaire signée en 2003 a créé le cadre pour l'organisation de la 
formation continue en interne à l'établissement avec pour éléments centraux la planification des qualifications requises dans l'établissement et la signature de conventions individuelles de qualification. Dans ces dernières sont fixés d'une part objectifs et contenus de la formation continue, d'autre part, l'apport individuel du salarié en termes de temps. La règle de base est celle d'un partage équitable des coûts entre salarié et employeur prenant en considération les avantages qu'en retire l'établissement aussi bien que le salarié à titre individuel (logique de co-investissement).

Les conventions tarifaires de ces branches-phares de l'industrie révèlent le rôle central dévolu au Conseil d'établissement dans le déclenchement comme l'organisation de programmes de formation continue. Dans les industries métallurgique et électrotechnique, leur mise en œuvre est de facto tributaire de la coopération du Conseil d'établissement, dans l'industrie chimique, la conclusion d'un accord d'établissement est même une condition expresse pour l'entrée en vigueur de la convention tarifaire. Mais les salariés eux aussi ont vu nettement renforcer, grâce à l'instrument qu'est la convention individuelle de qualification, le rôle qu'ils jouent au sein de leur établissement dans la gestion prévisionnelle individuelle et collective des compétences.

Le rôle important du Conseil d'établissement dans le processus des qualifications dans l'établissement repose par ailleurs sur la Loi sur la Constitution interne de l'entreprise (Betriebsverfassungsgesetz) qui lui confère des droits en matière d'initiative et de co-décision en ce qui concerne la formation continue interne à l'entreprise. Les dispositions de la loi portent essentiellement sur sa co-décision dans la mise en œuvre des actions de formation continue en interne offerte ou décidées par l'employeur. Le Conseil d'établissement peut donc négocier avec l'employeur sur les contenus et l'étendue des savoirs et savoir-faire à dispenser, sur les méthodes de formation, sur la durée et le calendrier de la formation continue, sur la délégation des formateurs, ainsi que sur le cercle des participants. La réforme de la Loi sur la Constitution interne de l'entreprise, intervenue en 2001 sous le gouvernement fédéral SDP/Verts, a étendu les prérogatives du Conseil d'établissement, lui donnant le droit d'exiger de la part de l'employeur que soit déterminé le besoin d'une hausse des qualifications dans l'établissement. De même, le Conseil peut formuler des recommandations sur le profil de qualification des salariés lors du planning des effectifs ou des mesures de préservation de l'emploi ; l'employeur est alors tenu d'engager des consultations avec le Conseil d'établissement. Enfin, ce dernier dispose en outre d'un pouvoir d'initiative préliminaire en la matière : il est en droit d'exiger des mesures de formation dans le cas où l'évolution des tâches fait que les compétences et savoir-faire des salariés ne sont plus adaptées. Si aucun accord ne peut être trouvé avec l'employeur, la décision est confiée en dernier ressort à une commission de conciliation chargée de résoudre le conflit, et composée de représentants des salariés et de l'employeur sous la présidence d'un expert externe, généralement un juge du travail.

Face à ces dispositions, un observateur externe pourrait avoir l'impression qu'en Allemagne, la formation continue en entreprise est fortement réglementée et relève de normes strictes. Mais il faut prendre en considération le fait que le cadre défini par les conventions tarifaires et le régime de la cogestion dans l'entreprise relève par essence d'une régulation procédurale, axée sur le process de production, et permettant de fixer les procédures de mise en œuvre de la formation en interne. On peut considérer cela comme une régulation flexible de la formation continue qui accorde la priorité à l'établissement. Toutefois, le recul du taux d'organisation des entreprises au niveau de la branche, de même que celui des établissements ayant une représentation élue des salariés (Betriebsrat) amène à relativiser quelque peu ce constat.

En Allemagne, ce sont les syndicats et les fédérations patronales de branche qui concluent les conventions tarifaires s'appliquant à l'ensemble de la branche et à l'échelle de toute une région ou de l'ensemble de l'Allemagne (Flächentarifverträge). Ces dernières années, la part des entreprises affiliées à une fédération
Rôle central du Conseil d'établissement...

... en application de la Loi sur la Constitution interne de l'entreprise

Une régulation flexible de la formation continue, aujourd'hui mise à mal par le recul du taux d'organisation 
L'entreprise n'en reste pas moins le premier offreur et organisateur de formation en interne
En comparaison internationale, l'Allemagne se situe dans la moyenne...

... en matière de formation continue sous forme de cours... patronale de branche a nettement reculé ; en reflet, la même tendance affecte le taux de syndicalisation des salariés dans les établissements. Au total, en Allemagne de l'est comme de l'ouest, le taux d'organisation a chuté dramatiquement entre 1996 et 2009 : depuis, seulement $32 \%$ des établissements et $52 \%$ des salariés sont directement couverts par une convention tarifaire de branche. II faut y ajouter cependant qu'un quart supplémentaire des établissements, occupant près d'un cinquième du total des salariés, se réfèrent volontairement aux conventions de branche, c'est-à-dire les appliquent alors qu'elles ne sont pas membres de la fédération de branche. Enfin, $3 \%$ des établissements (un dixième des salariés) ont conclu un accord d'entreprise (Firmentarifvertrag). C'est dans les nouveaux Länder et dans les établissements du secteur des transports, des TIC, comme des services marchands ou de la science, que le taux d'organisation est le plus faible.

Comme l'institution d'un Conseil d'établissement est certes un droit, mais non pas un devoir contraignant, la présence de cette représentation élue des salariés varie grandement selon la taille de l'établissement. Si la part des établissements où il en existe un stagne aux alentours de $10 \%$, dans le même temps, la part des salariés employés dans ces établissements a nettement diminué pour ne situer plus qu'à $45 \%$ du total à l'ouest et à $38 \%$ à l'est (2009). Ce recul s'observe principalement dans les entreprises de taille moyenne (50 à 500 salariés). A l'inverse, la part de celles de plus de 500 salariés reste supérieure à $90 \%$, alors qu'un peu plus de $10 \%$ seulement des petites entreprises (moins de 50 salariés) ont élu une représentation de leurs salariés.

Le recul du taux d'organisation comme de la densité des Conseils d'établissement relativise quelque peu l'importance de la régulation ou réglementation de la formation continue par les conventions tarifaires et la Loi sur la Constitution interne de l'entreprise. II n'en reste pas moins que, dans l'ensemble, les établissements en restent le principal offreur et organisateur, et que plus de salariés suivent une formation continue en interne plutôt qu'en externe.

\section{Formation interne à l'établissement : une stratégie de qualification insérée dans le process de production}

Comment se présente donc la réalité dans l'entreprise ? Dans les études comparées européennes, l'Allemagne se situe régulièrement dans la moyenne. Ainsi, l'enquête statistique européenne sur la formation continue CVTS (Continuing Vocational Training Survey), menée tous les six ans et dont la dernière vague remonte à 2005, révèle pour l'Allemagne des taux nettement inférieurs à ceux des autres économies industrielles européennes en ce qui concerne aussi bien l'implication des entreprises que la participation des salariés. Même si la diversité des méthodologies statistiques employées dans le cadre de l'enquête CVTS limite quelque peu la pertinence des données publiées et surtout laisse planer le doute sur l'exactitude du classement des pays, cela n'enlève rien au fait que la place moyenne occupée par l'Allemagne dans le contexte international est largement confirmée.

En ce qui concerne la formation continue sous la forme de cours, le taux de participation des entreprises allemandes était de $54 \%$ en 2005, ce qui les place loin derrière la Suède $(72 \%)$, la France $(71 \%)$, de même que les Pays-Bas et la Finlande (respectivement $70 \%$ ). Par rapport à 1999, ce taux a reculé de 13 points de pourcentage en Allemagne, de 12 aux Pays-Bas, de 11 en Suède et de 5 en Finlande, alors qu'il est resté constant en France. Quant au taux de participation des salariés à des cours de formation continue internes à l'entreprise, l'enquête CVTS révèle qu'il est passé de $32 \%$ à $30 \%$ en Allemagne entre 1999 et 2005. Dans d'autres pays nord- et ouest-européens, où ce taux a certes diminué plus fortement encore, mais en partant d'un niveau plus élevé, la participation des salariés n'en reste pas moins parfois nettement supérieure à ce qu'elle est en Allemagne. En France, le taux de participation (46\%) est resté constant. 
En matière d'actions de formation continue formalisées (cours), les données comparées révèlent donc une nette avance des entreprises françaises sur les allemandes. Mais la situation s'inverse lorsqu'on considère ce qu'il est convenu d'appeler les autres formes de formation continue : qualification sur le lieu de travail, initiation aux tâches, cercles de qualité, auto-formation classique et via l'e-learning. Alors, avec un taux de participation de $66 \%$, les entreprises allemandes ne sont certes qu'en deuxième position derrière l'Autriche (67 \%), mais elles mènent largement devant leurs homologues suédoises (60\%), finnoises (56\%), hollandaises (52\%) et françaises (44\%).

Ce constat incite à penser que la pratique de la formation continue dans les entreprises allemandes diffère de ce qu'elle est dans les autres pays. La différence d'approche semble liée à l'organisation de l'entreprise et du travail dans l'établissement (répartition des tâches, hiérarchies plates ou non, degré de responsabilité et d'autonomie des collaborateurs), de même qu'à la particularité du système de formation initiale des salariés (apprentissage vs. formation par la voie scolaire) qui entraîne par nature d'autres approches de la formation continue. Certes, l'enquête européenne CVTS ne fournit aucun élément d'explication. Mais force est de constater que, depuis ces dernières années, la qualification des salariés sur leur lieu de travail, dans l'objectif d'un développement permanent de leurs compétences au sein même des process de production, figure en Allemagne en tête des priorités de la formation continue interne à l'entreprise.

Pour mieux comprendre comment se présente, en Allemagne, la réalité dans l'entreprise, il faut recourir à une enquête du Zentrum für Sozialforschung de Halle ; bien que réalisée en 2002, elle n'en fournit pas moins des indications précieuses. Elle cherche en effet à savoir comment les entreprises organisent l'acquisition des savoirs nécessaires à l'optimisation de leurs process de production. Or dans le cadre du recrutement de leur personnel, un établissement a toujours plusieurs possibilités : proposer une formation initiale sous diverses formes, investir dans le perfectionnement des qualifications de ses salariés ou recruter les compétences requises sur le marché de l'emploi. Ceci posé, l'enquête distingue entre actions de formation continue formalisées, modes traditionnels d'acquisition informelle des compétences et nouveaux modes. Les actions formalisées désignent l'ensemble de l'offre se présentant sous la forme de cours, qu'ils soient organisés en interne ou en externe. Les modes traditionnels d'acquisition des compétences regroupent la formation continue sur le poste de travail, l'instruction par le supérieur hiérarchique, la participation à des conférences et séminaires spécialisées comme à des séminaires d'information. Enfin, les nouvelles formes d'acquisition informelle des savoirs rassemblent le travail en équipe, les cercles de qualité, le transfert des savoirs entre collègues, la mobilité interne (jobrotation), les partenariats, le coaching et l'e-learning.

L'enquête parvient à la conclusion que le nombre d'établissements mettant régulièrement en œuvre l'une de ces formes d'acquisition des savoirs et savoir-faire est bien plus grand que ce qu'on croyait jusqu'alors : $81 \%$ d'entre elles recourent à la formation formalisée, $92 \%$ aux méthodes traditionnelles d'acquisition informelle des compétences, et $74 \%$ aux nouvelles. Cela étant, la différenciation habituelle dans la participation à la formation continue selon le statut des salariés dans l'entreprise persiste à un haut niveau : les salariés non ou peu qualifiés ont toujours bien plus rarement accès à l'ensemble de la gamme des actions de formations que les ouvriers ou employés qualifiés. Ainsi, les peu ou non qualifiés sont $47 \%$ à participer à des actions formalisées, $64 \%$ aux modes informels traditionnels et $70 \%$ aux nouveaux. Pour les employés qualifiés, ces taux sont respectivement de $84 \% ; 91 \%$ et $93 \%$.

L'enquête menée par le Zentrum für Sozialforschung de Halle fait apparaître que ce qui détermine l'intensité comme les modes de l'action de formation dans l'entreprise, c'est d'une part le besoin de développer les compétences internes en accompagnement d'une stratégie d'innovation technique ou organisationnelle, et d'autre part les choix effectués parmi les divers modes de recrutement - interne
... mais elle figure en tête pour les autres formes... mais elle figure en tête pour les autres formes

Des raisons liées à l'approche de la compétitivité et au système dual

Une enquête menée en 2002 auprès des entreprises...

... révèle la place privilégiée des modes informels d'acquisition des compétences

Ils accompagnent les stratégies d'innovation 
Et des études de cas révèlent la priorité accordée aux compétences transversales..

... et à une formation tout au long de la vie qui est insérée dans le process de production

Mais les rigidités du système traditionnel d'éducation/formation brident la reconnaissance des savoirs acquis de manière informelle

Sous l'effet de la tertiarisation, l'apprentissage dual est en recul ou externe - du personnel. Or cette approche de la formation continue qui privilégie l'acte d'apprendre est nettement moins visible et quantifiable de l'extérieur que les actions traditionnelles de formation qui s'appuient sur la délivrance de savoirs formalisés.

L'importance croissante accordée au développement des compétences en interne se manifeste également à travers diverses études de cas menées dans les entreprises allemandes. Elles révèlent que, au cours des dernières années, les actions formalisées ont été fortement réduites en volume au profit des modes informels d'acquisition des savoirs, traditionnels et nouveaux. On observe la tendance à " mobiliser et développer les compétences extra-fonctionnelles grâce à une acquisition des savoirs peu ou pas formalisée " (Baethge-Kinsky, 2004). Les compétences professionnelles qui ne sont pas directement liées à l'activité exercée, mais sont plus générales et surtout transversales, gagnent donc en importance. Pourtant, les données de l'enquête européenne CVTS affirment que les modes informels d'acquisition des savoirs ne se substituent pas à la formation professionnelle traditionnelle, mais qu'elles ne viennent qu'en complément. L'enquête CVTS 2 (1999) révèle que ces " autres formes » de formation continue sont aussi souvent pratiquées dans les PME allemandes que celles sous forme de cours, alors que les entreprises françaises y recourent nettement moins souvent qu'aux « cours » ou séminaires (Behringer/Descamps, 2009). II s'ensuit que la participation des salariés allemands aux modes informels est aussi la plus élevée dans les entreprises qui offrent et mettent en œuvre des actions formalisées.

Une analyse de 22 études de cas sur la formation tout au long de la vie que nous avons menée dans le cadre de notre activité de conseil aux Conseils d'établissement au sein de la Fondation Hans Böckler retrace la pratique du développement plutôt informel des compétences au sein de l'établissement. Lorsque le développement des compétences se fait en interne, la formation tout au long de la vie prend alors la forme d'un processus qui, par nature, ne peut se contenter des savoirs cloisonnés délivrés sous forme de cours ou d'unités de formation, mais doit tenir compte des tâches confiées au salarié et s'insérer ainsi dans le process de production. Les actions de qualification pour adapter et développer les compétences indispensables à l'innovation sont généralement menées dans l'établissement, malgré quelques frictions. Les points faibles constatés concernent moins le développement des compétences liées aux fonctions exercées dans l'entreprise que les actions de formation destinées à améliorer l'employabilité et à répondre aux besoins individuels des salariés.

D'autres exemples encore montrent comment les processus d'apprentissage sur le poste de travail sont combinés avec des modes d'acquisition du savoir en dehors du travail et une offre de formation pour l'épanouissement individuel, et comment la combinaison de ces divers modes peut être mise à profit pour la préparation à un diplôme sanctionnant une action formalisée de formation professionnelle. Certaines dispositions contenues dans les accords d'entreprise ouvrent la possibilité, dans le cadre des entretiens de bilan, de faire valoir non seulement des objectifs d'optimisation des compétences sur le poste de travail, mais aussi des objectifs de développement personnel, et de procéder à la certification des savoirs acquis. Promouvoir l'aptitude à apprendre en même temps que l'investissement des salariés permet de garantir un développement continu des compétences. Toutefois, le système allemand de certification ne permet guère encore de sanctionner par un diplôme reconnu de formation professionnelle les parcours de qualification en interne modulés par étapes. Ce sont alors les dispositions réglementaires du système de formation/éducation traditionnel qui limitent la possibilité de faire reconnaître les compétences complémentaires acquises par le salarié en interne dans le système des parcours individualisés de développement des qualifications et de les y intégrer sous forme de modules.

L'organisation de l'acquisition des savoirs dans l'entreprise est en mutation ; de nouvelles formes de développement du personnel apparaissent, répondant aux exigences des nouveaux process de production. II faut alors considérer la for- 
mation continue en la resituant dans le contexte de la formation professionnelle initiale dans l'entreprise (apprentissage dans le système dual). Ce mode de formation joue certes encore un rôle important dans le débat sur la politique de formation et d'éducation, mais il a en grande partie perdu dans nombre d'entreprises le rôle prédominant qu'il a joué durant des décennies pour le recrutement et le développement des ressources humaines. Une des raisons en est plus particulièrement que dans les nouvelles branches nées avec la tertiarisation et l'avènement de l'économie du savoir, le système dual n'a jamais pu réellement s'installer.

C'est là le contexte dans lequel, à notre sens, il faut replacer les évolutions constatées au niveau de l'entreprise. D'une part, pour des raisons de coûts et de praticabilité (retour sur investissement), les contenus de la formation initiale sont de plus en plus déterminés par les compétences requises dans les process de production, et la formation sanctionnée de même. Cela signifie que, souvent, la formation des apprentis ne s'effectue plus dans un atelier d'apprentissage, mais sur le lieu de production, c'est-à-dire le futur poste de travail. D'autre part, les établissements commencent (avec quelques réticences encore toutefois) à adapter leur formation initiale et continue aux exigences du cadre européen des certifications (CEC) comme du futur système européen de crédit d'apprentissages pour l'enseignement et la formation professionnels (ECVET). Ensuite, les grandes entreprises organisent de plus en plus volontiers des cursus de formation professionnelle au-delà du niveau traditionnel de l'apprentissage dans le système dual, c'est-à-dire en coopération avec les universités et les "académies profesionnelles » (Berufsakademien) : des "cursus coopératifs » débouchant sur un bachelor et qui ouvrent de bonnes perspectives d'emploi. Enfin, ce sont majoritairement les grandes entreprises aussi qui organisent le renouvellement permanent des compétences de leurs cadres supérieurs et dirigeants sous la forme « d'académies » internes ou de "Corporate Universities » (celles-ci n'ayant en commun avec une université que le nom). Ces nouvelles formes d'organisation révèlent que les entreprises manifestent un besoin de développer les compétences humaines déterminé par leurs objectifs stratégiques de performance et axé sur le processus de leur mise en œuvre, autrement dit : leurs priorités dépassent ce que peut offrir le système dual traditionnel de formation initiale.

LE CONSTAT QUE, EN COMPARAISON INTERNATIONALE, participation et partie prenante à la formation continue formalisée soient faibles en Allemagne n'est pas resté sans conséquences sur le débat de politique d'éducation/formation. Toutes les représentations de la société civile et toutes les organisations politiques considèrent avec inquiétude les déficits perçus en la matière. Et, en fonction des intérêts politiques et organisationnels, on présuppose que la cause en réside tantôt dans le manque de motivation de l'individu ou du politique, tantôt dans le manque de structures ou leur développement insuffisant.

Les gouvernements fédéraux cherchent, quelle que soit leur obédience, à promouvoir la formation individuelle grâce au lancement d'une multitude de programmes, à intégrer l'offre des organismes de formation et à contribuer à l'amélioration de leur qualité. Toutes ces mesures doivent respecter un délicat équilibre entre objectifs politiques, répartition des compétences législatives au sein du fédéralisme et la prééminence de la responsabilité des entreprises pour la formation continue en interne.

En 2004, une commission d'experts pour le financement de la formation tout au long de la vie avait formulé pour le compte du gouvernement fédéral SPD/Verts une série de recommandations dont le résultat devait être de constituer un système cohérent en la matière. Le gouvernement de Grande coalition qui lui succéda a tenté, via l'institution de "Cercles d'innovation » (Innovationskreise), composés de représentants de haut niveau des entreprises, des partenaires sociaux et du monde de la recherche, de lancer des évolutions consensuelles sur de nombreux aspects particuliers liés à la formation tout au long de la vie. La
Le système dual

n'est plus adapté à la nouvelle demande des entreprises 
plupart des projets et recommandations sont cependant restés plutôt vagues, s'assimilant à une approche programmatique. Seuls quelques éléments isolés furent mis en œuvre. II s'agit principalement de l'extension de la loi fédérale relative à la promotion des formations de perfectionnement (Meister-BAFöG) à d'autres diplômes professionnels et de la création d'une " prime de formation " (Bildungsprämie ; $500 €$ par an au maximum) versée en soutien à l'épargne de formation individuelle. Toutes les mesures des gouvernements fédéraux dans ce domaine ont laissé en l'état les structures traditionnelles de la formation continue, toujours aussi peu développées. A l'opposé, l'annonce de campagnes publiques pour promouvoir la motivation à suivre une formation et la création "d'alliances pour la formation » (Bildungsallianzen) réunissant partenaires sociaux et autres acteurs de formation ont eu davantage d'effet ; et on espère qu'elles créeront une dynamique d'auto-promotion des « bonnes pratiques » dans le domaine de la formation continue.

Pour leur part, les syndicats allemands préfèrent, tant au niveau des programmes que de l'action au quotidien, que soient prises des dispositions légales contraignantes pour la réglementation et, plus encore, pour le financement de la formation continue. Dans leur approche, les ressources clefs qui demandent à être mieux développées pour diffuser la formation continue sont le temps et l'argent. Et leur sympathie va justement aux structures de fonds de financement cogérés par les partenaires sociaux, tels qu'elles se sont développées en France. En effet, ces structures, argumentent-ils, ont permis sur le long terme que la participation des salariés soit stable et d'un niveau plus élevé qu'en Allemagne, parce que, surtout pour les petites entreprises, elles allègent les coûts financiers de la formation ou les prennent à leur charge.

Alors que dans cette interprétation syndicale du dispositif français, les dispositions adoptées au niveau collectif, c'est-à-dire dépassant le cadre d'un établissement isolé, contribuent à la diffusion de la formation continue et, le cas échéant, soutiennent le développement des compétences individuelles, la situation allemande est marquée par la prédominance d'une démarche individualisée de l'entreprise qui peut, sur le long terme, se révéler dommageable à l'employabilité Après tout, dans son rapport 2007 sur l'éducation ("Education at a Glance »), I'OCDE avance l'hypothèse que le risque croissant avec l'âge (au-delà de 50 ans) de se trouver au chômage que rencontrent les bacheliers ou les sortants du système dual, à la différence des diplômés de l'enseignement supérieur (université et Fachhochschule), serait dû à la faible adaptabilité des premiers aux exigences du marché de l'emploi. En d'autres termes : si l'insertion dans la vie professionnelle des sortants du système d'apprentissage dual se fait sans grands heurts, leur risque d'être exposés au chômage va croissant avec l'âge (Schleicher, 2007). Et cela pourrait être lié, selon une autre hypothèse, à la pratique de la formation continue majoritairement suivie en interne, ce qui tendrait à réduire l'adaptabilité.

On peut toutefois émettre l'hypothèse fondée que les modes et pratiques spécifiques des entreprises allemandes en matière de stratégies de qualification, conjugués au processus de rationalisation interne mené dans les établissements au cours des dernières années et à la conclusion de " Pactes de modernisation et de préservation de l'emploi » au sein de ces derniers dans la tradition du système de cogestion, sont une raison décisive expliquant le succès des entreprises allemandes à l'export. La question des déséquilibres au sein de l'économie européenne ne peut en aucun cas, contrairement à ce que laissent penser les débats actuels, être traitée dans une perspective purement macroéconomique ni se réduire à une alternative opposant politique économique axée sur la demande intérieure et politique économique tournée vers l'export.

Trouver un équilibre entre d'un côté une formation continue formalisée dispensant une employabilité durable dans une économie en mutation et, de l'autre, un développement des compétences orienté sur les process de production et axé sur l'innovation comme la productivité de l'entreprise - voilà le défi que doit relever la politique allemande de la formation continue. Les conventions tarifaires 
peuvent créer un cadre qu'il revient au dirigeants et au Conseil d'établissement de concrétiser par la conclusion d'accords d'entreprise. Cette solution aurait le mérite de ménager la particularité et les avantages spécifiques des relations industrielles en Allemagne et d'éviter de tomber dans le piège d'une réglementation trop rigide. Cela étant, l'expérience a montré que, dans leur mise en pratique, les conventions tarifaires conclues en ce sens se révélaient plutôt ambivalentes: si elles n'ont pas contribué à une hausse perceptible de la participation à la formation continue en interne, elles n'en ont pas moins permis de développer une culture de la responsabilité en la matière, tout particulièrement via l'outil d'adaptation et de modernisation des compétences requises au niveau de l'établissement que sont les bilans de qualification. Certains, dans le syndicalisme allemand, considèrent avec sympathie la législation française relative à la formation continue étant donné que, y compris au regard de l'enquête CVTS, ses dispositions sont indéniablement efficaces pour générer des actions de formation continue formalisée par des cours. II ne faut cependant pas perdre de vue que ces dispositions sont moins propices aux modes non formalisés de formation continue (développement informel des compétences) et qu'elles ont une portée moindre en ce qui concerne la formation continue dans les petites entreprises (Behringer/ Descamps).

Traduction : I. Bourgeois

\section{Indications bibliographiques}

- BAETHGE-KINSKY V., « Zur Entwicklungsdynamik nicht formalisierter Lernprozesse - Qualifizierungsziele und Probleme », in MEIER Heike (ed), Kompetenzentwicklung in deutschen Unternehmen. Formen, Voraussetzungen und Veränderungsdynamik, in Forschungsberichte aus dem zsh 04-3, Halle, 2004

- BehRinger F., Descamps R., « Determinants of employer-provided training : A comparative analysis of Germany and France ", in BeHRINGER F., KäPPLINGER B., PÄTZOLD G. (eds), Betriebliche Weiterbildung - der Continuing Vocational Training Survey (CVTS) im Spiegel nationaler und europäischer Perspektiven, in Zeitschrift für Berufs- und Wirtschaftspädagogik, Beiheft 22, Stuttgart, 2009

- Heidemann W. (ed), Lebenslanges Lernen im Betrieb - Neuere Praxisbeispiele, Arbeitspapier der Hans-Böckler-Stiftung, $n^{\circ} 153$, Düsseldorf, 2010

- LASSERRe R., ZetTelmeier W., Etude du système de formation professionnelle en Allemagne, réalisée pour la Cour des Comptes ( $5^{e}$ Chambre), CIRAC, Cergy-Pontoise, 2007

- SCHLeICHER A., Bildung auf einen Blick 2007 - OECD Briefing Notes für Deutschland, publication électronique, http://www.oecd.org/dataoecd/34/39/39284171.pdf (consultée le 05-05-2010)

- WINGE S., WIENER B., Lernen in kleinen und mittleren Unternehmen, in Forschungsberichte aus dem zsh 09-1, 2009. 